GUIDELINES

\title{
Diagnosis and management of adults with chronic heart failure: summary of updated NICE guidance
}

\author{
Abdallah Al-Mohammad, Jonathan Mant, ${ }^{1}$ Philippe Laramee, ${ }^{3}$ Sharon Swain, ${ }^{3}$ on behalf of the Chronic Heart \\ Failure Guideline Development Group
}

\begin{abstract}
1South Yorkshire Cardiothoracic
Centre, Sheffield Teaching

Hospitals NHS Foundation Trust, Sheffield S57AU

${ }^{2}$ General Practice \& Primary

Care Research Unit, University of

Cambridge CB2 OSR

${ }^{3}$ National Clinical Guideline Centre,

Royal College of Physicians of

London, London NW14LE

Correspondence to:

A Al-Mohammad

a.al.mohammad.87

@googlemail.com
\end{abstract}

Cite this as: $B M J$ 2010;341:C4130 doi: 10.1136/bmi.c4130

This is one of a series of $B M J$ summaries of new guidelines based on the best available evidence; they highlight important recommendations for clinical practice, especially where uncertainty or controversy exists.

Further information about

the guidance, including the supporting evidence statements and the members of the Guideline Development Group, is in the full version on bmj.com.
Heart failure affects 900000 people in the United Kingdom. ${ }^{1}$ Its prevalence is increasing owing to improved prognosis of ischaemic heart disease (the major cause of heart failure) and an ageing population. ${ }^{2}{ }^{3}$ The two main types of heart failure are left ventricular systolic dysfunction and that associated with preserved left ventricular ejection fraction. Both types have a poor prognosis, although the introduction of effective treatments has led to a fall in mortality from heart failure caused by left ventricular systolic dysfunction (from $26 \%$ at six months in 1995 to $14 \%$ at six months in 2005). ${ }^{4}$ New evidence has emerged on diagnosis, treatment, rehabilitation, and monitoring of people with heart failure, and use of this evidence to guide diagnosis and management is likely to improve outcomes further and increase the cost effectiveness of services. This article summarises the most recent recommendations from the National Institute for Health and Clinical Excellence (NICE) on the diagnosis and management of chronic heart failure (which is a partial update of its 2003 guidelines $\left.{ }^{5}\right)^{6}$

\section{Recommendations}

NICE recommendations are based on systematic reviews of best available evidence and explicit consideration of cost effectiveness. When minimal evidence is available, recommendations are based on the Guideline Development Group's experience and opinion of what constitutes good practice. The new recommendations are indicated in parentheses. Evidence levels for the recommendations are in the full version of this article on bmj.com.

With the exception of the recommendations on drug treatment, all the recommendations apply to the diagnosis and management of heart failure with left ventricular systolic dysfunction and heart failure with preserved ejection fraction.

\section{Diagnosis}

- In patients with suspected heart failure and previous myocardial infarction, refer urgently for transthoracic Doppler two dimensional echocardiography and specialist assessment (both to take place within two weeks of referral). (New recommendation.)

- In patients with suspected heart failure and without previous myocardial infarction, measure serum natriuretic peptides (B-type natriuretic peptide or $\mathrm{N}$-terminal pro-B-type natriuretic peptide) levels. (New recommendation.)
-If the B-type natriuretic peptide level is $>400$ $\mathrm{pg} / \mathrm{ml}$ (116 pmol/l) or an N-terminal pro-Btype natriuretic peptide level is $>2000 \mathrm{pg} / \mathrm{ml}$ (236 pmol/l), refer urgently for transthoracic Doppler two dimensional echocardiography and specialist assessment (both to take place within two weeks of referral) as very high levels of serum natriuretic peptides carry a poor prognosis. (New recommendation.)

-If the B-type natriuretic peptide level is 100-400 $\mathrm{pg} / \mathrm{ml}$ (29-116 pmol/l), or an N-terminal pro-Btype natriuretic peptide level is $400-2000 \mathrm{pg} / \mathrm{ml}$ (47-236 pmol/l), refer for transthoracic Doppler two dimensional echocardiography and specialist assessment (both to take place within six weeks of referral). (New recommendation.)

-If the serum B-type natriuretic peptide level is $<100 \mathrm{pg} / \mathrm{ml}$ (29 pmol/litre) or an N-terminal proB-type natriuretic peptide level $<400$ pg/ml (47 $\mathrm{pmol} /$ litre), a diagnosis of heart failure is unlikely in an untreated patient. (New recommendation.)

- Situations that may reduce serum natriuretic peptide levels include obesity or treatment with diuretics, angiotensin converting enzyme (ACE) inhibitors, angiotensin receptor blockers, $\beta$ blockers, or aldosterone antagonists. (New recommendation.)

- Conditions other than heart failure that may raise serum natriuretic peptide levels include diabetes, sepsis, age $>70$ years, and those affecting the heart (left ventricular hypertrophy, myocardial ischaemia, tachycardia, right ventricular overload); the lungs (hypoxaemia-from causes including pulmonary embolism and chronic obstructive pulmonary disease); the kidneys (glomerular filtration rate $<60 \mathrm{ml} / \mathrm{min}$ ); and the liver (cirrhosis). (New recommendation.)

- Do electrocardiography and consider tests to evaluate possible aggravating factors and/or alternative diagnoses. The tests include chest radiography, pulmonary function tests, urine analysis, and blood tests for full blood count and film plus biochemical assessment of the liver, kidneys, thyroid, glucose, and lipids.

- Once heart failure has been diagnosed, assess severity, aetiology, precipitating factors, type of 


\section{New York Heart Association (NYHA) classification of heart} failure symptoms

Class I-No limitations

Class II-Slight limitation of physical activity

(symptomatically “mild” heart failure)

Class III-Marked limitation of physical activity

(symptomatically "moderate" heart failure)

Class IV - Symptoms of heart failure are present even at rest (symptomatically "severe" heart failure)

cardiac dysfunction (whether it is associated with left ventricular systolic dysfunction or preserved left ventricular ejection fraction), and correctable causes. (New recommendation.)

\section{Referral and approach to care}

- Care should be delivered by a multidisciplinary team with an integrated approach across the healthcare community.

- Refer patients to a specialist multidisciplinary heart failure team for initial diagnosis of heart failure and management of the following: severe heart failure (New York Heart Association (NYHA) class IV (see box defining the four classes)); heart failure that does not respond to treatment; heart failure resulting from valvular disease; and heart failure that can no longer be managed effectively in the home setting. (New recommendation.)

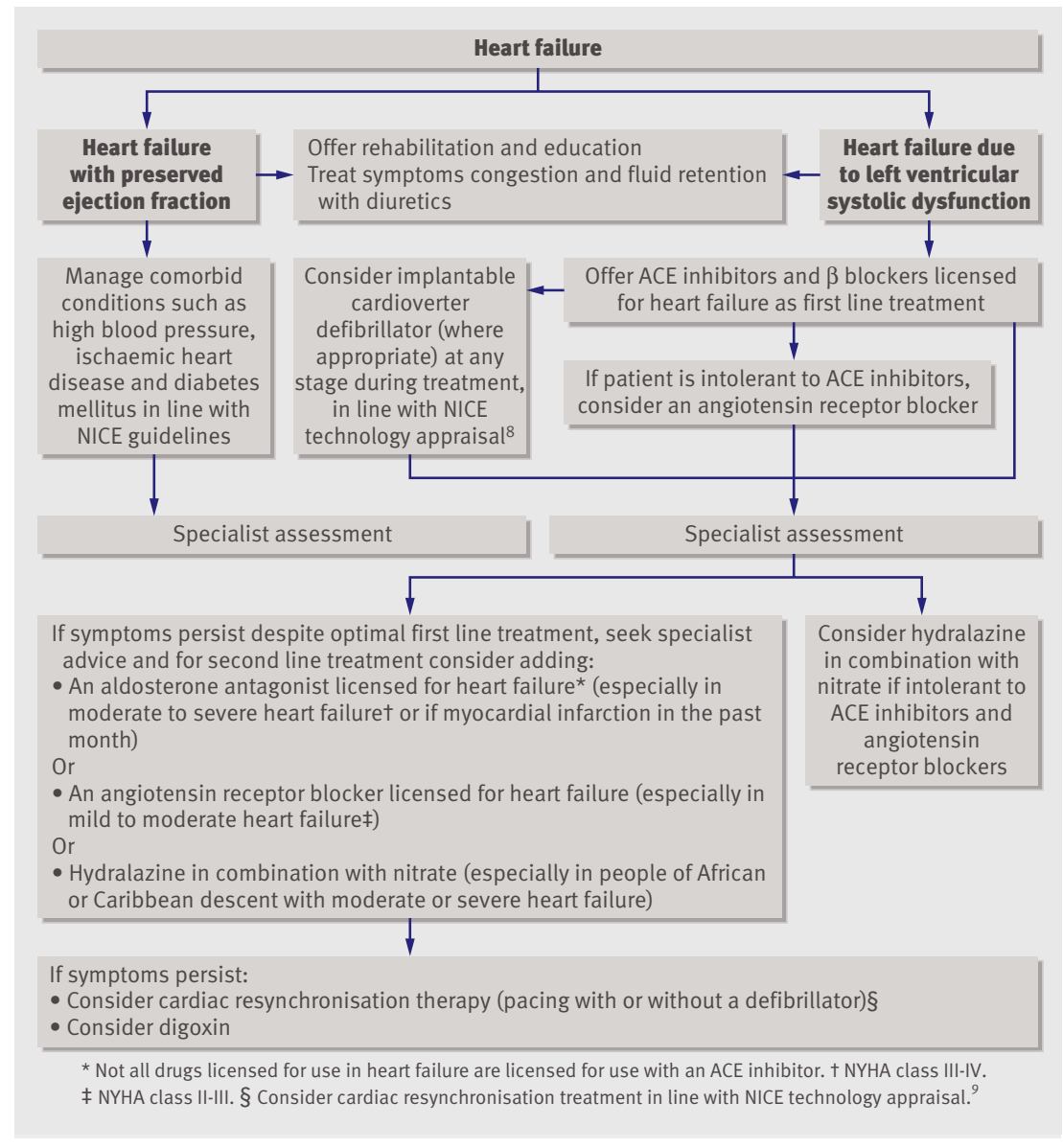

Treatment of heart failure with left ventricular systolic dysfunction

- Offer both ACE inhibitors and $\beta$ blockers licensed for heart failure to all patients with heart failure that is caused by left ventricular systolic dysfunction. (New recommendation.) With regard to $\beta$ blockers, this includes older adults as well as patients with peripheral vascular disease, erectile dysfunction, diabetes mellitus, interstitial pulmonary disease, or chronic obstructive pulmonary disease without reversibility. (New recommendation.)

-For patients who have intolerable side effects with ACE inhibitors, consider an angiotensin receptor blocker licensed for heart failure as an alternative. (New recommendation.)

-For patients who are intolerant of both ACE inhibitors and angiotensin receptor blockers, seek specialist advice and consider hydralazine in combination with nitrate. (New recommendation.)

- If a patient remains symptomatic despite optimal treatment with an ACE inhibitor and a $\beta$ blocker, consider adding second line treatment:

-An aldosterone antagonist licensed for heart failure, especially if the patient has moderate to severe heart failure (NYHA class III or IV) or has had a myocardial infarction within the past month. (New recommendation.) or -An angiotensin receptor blocker licensed for heart failure, especially if the patient has mild to moderate heart failure (NYHA class II or III). (New recommendation.) or

-Hydralazine in combination with nitrate (especially if the patient is of African or Caribbean descent with moderate to severe heart failure (NYHA class III or IV)). (New recommendation.)

- Consider digoxin for worsening or severe heart failure caused by left ventricular systolic dysfunction despite first line and second line treatment for heart failure.

\section{Practical prescribing recommendations}

- ACE inhibitors: Start at a low dose and titrate upwards at short intervals (for example, every two weeks) until the optimal tolerated or target dose is achieved. Measure serum urea, creatinine, electrolytes, and estimated glomerular filtration rate when starting an ACE inhibitor and after each dose increment.

- $\beta$ blockers: Start in a "start low, go slow" manner, and assess heart rate, blood pressure, and clinical status after each titration. If patients who are stable and already taking a $\beta$ blocker for a comorbidity (for example, angina or hypertension) develop heart failure caused by left ventricular systolic dysfunction, switch them to a $\beta$ blocker licensed for heart failure. (New recommendation.)

- Angiotensin receptor blockers: In patients taking these, monitor serum urea, electrolytes, creatinine, and estimated glomerular filtration rate for signs of renal impairment or hyperkalaemia. (New recommendation.) 


\author{
bmj.com archive \\ Previous articles in this \\ series \\ - Diagnosis, prevention, \\ and management \\ of delirium (BM) \\ 2010;341:c3704) \\ - Management of \\ bacterial meningitis \\ and meningococcal \\ septicaemia in children \\ and young people (BM) \\ 2010;340:c3209) \\ - Diagnosis and \\ clinical management of \\ alcohol related physical \\ complications (BMJ \\ 2010;340:c2942)
}

- Aldosterone antagonists: In patients taking these, closely monitor potassium, creatinine, and estimated glomerular filtration rate. Seek specialist advice if the patient develops hyperkalaemia or if renal function deteriorates. (New recommendation.)

Treatment of heart failure with preserved ejection fraction Treat congestive symptoms with diuretics, and aim for optimal management of comorbidities such as hypertension, ischaemic heart disease, and diabetes. Insufficient evidence exists to make a recommendation on the role of ACE inhibitors, angiotensin receptor blockers, and $\beta$ blockers in heart failure with preserved ejection fraction.

\section{Rehabilitation}

- Offer a supervised, exercise based, group rehabilitation programme designed for patients with heart failure in patients who are stable and do not have a condition or device that would preclude an exercise based rehabilitation programme (such as uncontrolled ventricular response to atrial fibrillation; uncontrolled hypertension; high energy pacing devices set for activation at rates likely to be achieved during exercise). Include a psychological and educational component in the programme. The programme may be incorporated within an existing cardiac rehabilitation programme. (New recommendation.)

\section{Monitoring}

- Seek the opinion of a specialist in heart failure to guide the care of patients admitted to hospital. (New recommendation.)

- Monitor all patients with chronic heart failure, including clinical assessment of functional capacity, fluid status, cardiac rhythm (minimum of examining the pulse), cognitive status, and nutritional status; medication review; and measurement of serum urea, creatinine, and electrolyte levels, and estimated glomerular filtration rate.

- Consider specialist monitoring of serum natriuretic peptides in some patients (such as those in whom uptitration of treatment is difficult, and those with previous admissions to hospital). (New recommendation.)

- The frequency of monitoring depends on the clinical status (including the presence of comorbidity) and stability of the patient. The monitoring interval should be short (from a few days to two weeks) if the clinical condition or medication has changed. Monitor stable patients at least every six months.

- Provide patients who wish to be involved in the monitoring of their condition with sufficient education and support from their healthcare professional to do this.

\section{Discharge planning}

- Patients should generally be discharged from hospital only when their clinical condition is stable and the management plan is optimised. Timing of discharge should take into account patients' and carers' wishes and the level of care and support that can be provided in the community. The primary care team, patient, and carer must be aware of the management plan. Give clear instructions on how the patient or carer can access advice, particularly in the high risk period immediately after discharge.

\section{Overcoming barriers}

Local access to natriuretic peptide testing, two dimensional transthoracic echocardiography, and specialist opinion will need to be improved for optimal implementation of the diagnostic pathway. Reduced emergency admissions as a result of earlier diagnosis will offset the implementation costs. Better uptake of proved interventions will depend on the training of staff who provide heart failure care in both primary and secondary care and better communication between generalists and specialists. Greater availability of rehabilitation programmes with a focus on heart failure will be necessary to ensure that more people have access to such programmes.

Contributors: AAI-M and JM drafted the article. All authors revised it critically for important intellectual content and approved the final version to be published. All authors are guarantors.

Funding: The National Clinical Guideline Centre was commissioned and funded by the National Institute for Health and Clinical Excellence to write this summary.

Competing interests: All authors have completed the Unified Competing Interest form at www.icmje.org/coi_disclosure.pdf (available on request from the corresponding author) and declare that (1) All of the authors have no financial support for the submitted work from anyone other than their employers; (2) In the previous three years AAl-M has been an investigator in the recently stopped Emphasis trial. AAl-M is a clinical adviser to the Health Technology Assessment programme of the National Institute for Health Research on the clinical and cost effectiveness of home telemonitoring or structured telephone support programmes for patients with severe chronic heart failure. AAl-M accepted in March 2008 hospitality from Novartis to attend the American College of Cardiology scientific meeting in Chicago, USA; the other authors declare no relationships (3) All authors have no spouses, partners, or children with financial relationships that may be relevant to the submitted work; and (4) all authors were members of the Guideline Development Group for the NICE guideline (AAl-M the clinical adviser, JM the chair, SS the systematic reviewer, and PL the health economist). The authors have no other nonfinancial interests that may be relevant to the submitted work.

Provenance and peer review: Commissioned; not externally peer reviewed.

1 Petersen S, Rayner M, Wolstenholme J. Coronary heart disease statistics: heart failure supplement. British Heart Foundation, 2002.

2 Cowie MR, Wood DA, Coats AJS, Thompson SG, Poole-Wilson PA, Suresh V, et al. Incidence and aetiology of heart failure: a populationbased study. Eur Heart J 1999;20:421-8.

3 Owan TE, Hodge DO, Herges RM, Jacobson SJ, Roger VL, Redfield MM. Trends in prevalence and outcome of heart failure with preserved ejection fraction. N Engl J Med 2006;355:251-9.

4 Mehta PA, Dubrey SW, McIntyre HF, Walker DM, Hardman SMC, Sutton $\mathrm{GC}$, et al. Improving survival in the 6 months after diagnosis of heart failure in the past decade: population-based data from the UK. Heart 2009;95:1851-6.

5 National Institute for Clinical Excellence. Chronic heart failure: management of chronic heart failure in adults in primary and secondary care (Clinical guideline 5). 2003. www.nice.org.uk/CG5

6 National Institute for Health and Clinical Excellence. Chronic heart failure: the management of adults with chronic heart failure in primary and secondary care (partial update). (Clinical guideline 108). 2010. www.nice.org.uk/CG108.

7 Guyatt GH, Oxman AD, Vist G, Kunz R, Falck-Ytter Y, Alonso-Coello P, et al. GRADE: an emerging consensus on rating quality of evidence and strength of recommendations. BMJ 2008;336:924-6.

8 National Institute for Health and Clinical Excellence. Arrhythmiaimplantable cardioverter defibrillators (ICDs) (review). (Technology appraisal 95.) 2006. http://guidance.nice.org.uk/TA95.

9 National Institute for Health and Clinical Excellence. Heart failurecardiac resynchronisation. (Technology appraisal 120.) 2007. http:// guidance.nice.org.uk/TA120. 


\title{
UNCERTAINTIES PAGE Should treatment for heart failure with preserved ejection fraction differ from that for heart failure with reduced ejection fraction?
}

\author{
Philip Jong, Robert McKelvie, Salim Yusuf
}

Population Health Research Institute, Hamilton Health Sciences, McMaster University, Hamilton,

Canada

Correspondence to: S Yusuf yusufs@mcmaster.ca

Cite this as: BMJ 2010;341:c4202 doi: 10.1136/bmj.c4202

This is one of a series of occasional articles that highlight areas of practice where management lacks convincing supporting evidence. The series advisers are Rubin Minhas, clinical director, BMJ Evidence Centre, and David Tovey, editor in chief, the Cochrane Library.
Heart failure with preserved ejection fraction (HF-PEF) accounts for about half of all cases of heart failure. ${ }^{1} \mathrm{Popu}-$ lation based studies have shown that patients with HF-PEF have high rates of mortality (20-30\% risk at one year) ${ }^{2}{ }^{3}$ and readmission to hospital (30\% risk at 60-90 days), ${ }^{4}$ which are similar to the rates for patients with heart failure with reduced ejection fraction (HF-REF). When compared with patients with HF-REF, those with HF-PEF are older, more often female, and more likely to have hypertension and atrial fibrillation, but are less likely to have coronary artery disease. ${ }^{2}{ }^{3}$ Identifying patients with HF-PEF can be difficult, however, because heart failure presents in many different ways and the diagnostic criteria that define this syndrome are complex. ${ }^{5}$ The guideline from the National Institute for Health and Clinical Excellence has proposed detailed guidance on diagnosis. ${ }^{6}$ In practice, the diagnosis of HF-PEF is often made in patients with symptoms and signs of heart failure who have preserved left ventricular ejection fraction. Furthermore, the evidence that supports treatment for HF-PEF is much weaker than that supporting treatment for HF-REF. To date, results from existing clinical trials of HF-PEF have been largely inconclusive, and treatments that have been shown to reduce morbidity and mortality in patients with HF-REF showed either no or only marginal benefits in patients with HF-PEF.

\section{What is the evidence of the uncertainty? \\ To clarify whether treatments that are beneficial in HF-REF may also be beneficial in HF-PEF, we searched Medline, Embase, and the Cochrane Library for all adequately powered randomised controlled trials of treatments for chronic heart failure (HF-PEF or HF-REF) that had reported both mortality and admission to hospital as their primary outcomes. We found no meta-analyses of treatments for HF-PEF, so we depended on studies that would provide the most robust and unbiased estimates of the tested treatments by selecting trials that had the largest sample size or the long- est follow-up and those that reported high quality outcome data, such as blinded adjudicated events.}

The table compares the degree of benefit of common treatments in HF-REF and in HF-PEF. To date, no drug or non-drug treatment has been shown to reduce mortality (all cause or cardiovascular) in patients with HF-PEF. By contrast, among patients with HF-PEF, some drug treatments have been shown to reduce admission to hospital for reasons related to heart failure.

\section{Randomised controlled trials of HF-PEF}

The most robust data are from trials that evaluated angiotensin converting enzyme (ACE) inhibitors and angiotensin receptor blockers. For ACE inhibitors, one trial showed over 26 months that in elderly patients with HF-PEF and an ejection fraction of $\geq 40 \%$, perindopril when compared with placebo did not reduce the composite outcome of all cause death or unplanned admissions to hospital for heart failure (hazard ratio 0.92; 95\% confidence interval 0.70 to 1.21$).{ }^{7}$ Moreover, the observed reduction at one year in admissions to hospital for heart failure with perindopril $(0.63 ; 0.41$ to 0.97$)$ was not sustained by the end of the trial $(0.86 ; 0.61$ to 1.20$)$, possibly owing to high rates of treatment crossover. However, perindopril reduced the severity of symptoms of heart failure.

For angiotensin receptor blockers, one trial showed over 37 months that in patients with HF-PEF and an ejection fraction of $>40 \%$, candesartan when compared with placebo did not reduce the composite outcome of cardiovascular death or admission to hospital for heart failure $(0.89 ; 0.77$ to 1.03). ${ }^{8}$ However, candesartan reduced admissions to hospital for heart failure alone by $16 \%$ (adjusted hazard ratio $0.84 ; 0.70$ to 1.00 ). In the same trial, patients receiving candesartan also showed less worsening of symptoms of heart failure. ${ }^{9}$ Another trial showed over 50 months that in patients with HF-PEF and an ejection fraction of $\geq 45 \%$, irbesartan when compared with placebo did not reduce the composite outcome of all cause death or admission to hospital for cardiovascular causes (hazard ratio $0.95 ; 0.86$ to 1.05$).^{10}$ Unlike candesartan, irbesartan did not reduce admissions

Qualitative summary of treatment effects of common treatments used in heart failure with reduced ejection fraction and in heart failure with preserved ejection fraction*

\begin{tabular}{|c|c|c|c|c|c|c|}
\hline \multirow[b]{2}{*}{ Treatment } & \multicolumn{3}{|c|}{ Heart failure with reduced ejection fraction } & \multicolumn{3}{|c|}{ Heart failure with preserved ejection fraction } \\
\hline & Mortality & Admission to hospital & Symptom & Mortality & Admission to hospital & Symptom \\
\hline ACE inhibitor & Substantial benefit & Substantial benefit & Marginal benefit & No benefit & Marginal or no benefit & No benefit \\
\hline Angiotensin receptor blocker & Marginal benefit & Substantial benefit & Marginal benefit & No benefit & Marginal or no benefit & No benefit \\
\hline$\beta$ Blocker & Substantial benefit & Substantial benefit & Marginal benefit & No benefit & No benefit & Marginal or no benefit \\
\hline Digitalis & No benefit & Substantial benefit & Marginal benefit & No benefit & No benefit & No data \\
\hline Aldosterone antagonist & Substantial benefit & Substantial benefit & No benefit & No data & No data & No data \\
\hline Cardiac resynchronisation therapy & Marginal benefit & Substantial benefit & Marginal benefit & No data & No data & No data \\
\hline Implantable cardioverter defibrillator & Substantial benefit & No benefit & No benefit & No data & No data & No data \\
\hline
\end{tabular}

${ }^{\star}$ The treatment effects are summarised qualitatively on the basis of the totality of currently available evidence rather than data from individual trials. 
to hospital for worsening heart failure ( $0.95 ; 0.81$ to 1.10 ). Irbesartan also did not reduce the severity of symptoms of heart failure or functional limitation.

For digitalis, an ancillary arm of one trial showed over 37 months that in patients with HF-PEF and an ejection fraction of $>45 \%$, digoxin when compared with placebo did not reduce the composite outcome of death from heart failure and admission to hospital $(0.82 ; 0.63$ to 1.07$)$ or admission to hospital alone for worsening heart failure $(0.79 ; 0.59$ to 1.04). ${ }^{11}$ Though not statistically significant, these results were consistent with those in the main arm of the trial, ${ }^{12}$ which showed a reduction in admissions to hospital for worsening heart failure in patients with HF-REF who were taking digoxin (risk ratio $0.72 ; 0.66$ to 0.79 ).

For $\beta$ blockers, one trial showed over 21 months that in the subgroup of elderly patients with an ejection fraction of $>35 \%$ (a cut-off value lower than that typically used for defining HF-PEF), nebivolol when compared to placebo did not reduce the composite outcome of all cause death or admission to hospital for cardiovascular causes (hazard ratio $0.82 ; 0.63$ to 1.05$).{ }^{13}$

No large trial has yet evaluated the role of aldosterone antagonists, direct vasodilators, calcium channel blockers, antiarrhythmics, antiplatelets, anticoagulants, cardiac resynchronization treatments, or implantable cardioverter defibrillators in patients with HF-PEF.

It is notable that, although trials of ACE inhibitors, angiotensin receptor blockers, digitalis, and $\beta$ blockers did not show a significant reduction in their primary outcomes, most found a non-significant reduction consistent with their effects in HF-REF. Therefore, when making treatment decisions, one approach is to infer from these data that these treatments may provide some benefit in patients with HF-PEF but less than in patients with HF-REF.

\section{Extrapolations from trials with preserved ejection fraction and no heart failure}

Another approach is to extrapolate from data in which the same treatment for "allied" conditions has been shown to prevent morbidity and mortality that are related to heart failure. These allied conditions include those that are risk factors for developing heart failure, such as hypertension and coronary artery disease, as well as those that are associated with heart failure, such as atrial fibrillation.

The best data come from trials that evaluated ACE inhibitors, angiotensin receptor blockers, and to a lesser extent $\beta$ blockers. In these trials, patients without heart failure mostly had preserved ejection fraction. For ACE inhibitors, three trials, of ramipril, perindopril, and trandolapril, all showed a reduction in adverse outcomes related to heart failure when compared with placebo. ${ }^{14} \mathrm{~A}$ combined analysis of these data showed that in patients with a wide spectrum of cardiovascular risks, ACE inhibitors lowered the risk of admission to hospital because of heart failure by $23 \%$ (hazard ratio $0.77 ; 0.67$ to 0.90 ).${ }^{14}$ For angiotensin receptor blockers, another trial showed that in patients with atrial fibrillation and other risk factors for stroke, irbesartan reduced admissions to hospital for heart failure by $14 \%$ (0.86; 0.76-0.98) when compared with placebo. ${ }^{15}$ For $\beta$ blockers, pooled trial data in patients with hypertension showed a trend in reduction in the risk of developing heart failure with $\beta$ blockers compared with placebo (risk ratio $0.77 ; 0.60$ to 1.01$).{ }^{16}$ As most patients with HF-PEF have one of these allied conditions before developing heart failure, it is reasonable in the absence of direct evidence to infer that such treatments (blockers of the renin-angiotensin-aldosterone system or $\beta$ blockers) may also benefit patients with HF-PEF.

\section{Is ongoing research likely to provide relevant evidence?}

A search of ClinicalTrials.gov (www.clinicaltrials.gov) and the International Clinical Trials Registry (apps.who. int/trialsearch) did not identify any ongoing large trials of HF-PEF evaluating ACE inhibitors, angiotensin receptor blockers, or $\beta$ blockers.

However, a multicentre, international, randomised, double blind placebo controlled trial of aldosterone antagonist treatment (the TOPCAT trial, www.topcatstudy.com/) is under way as follows:

- Population: Planned recruitment of about 4500 adults with HF-PEF, an ejection fraction of $\geq 45 \%$, and either a history of admission to hospital because of heart failure within the past 12 months or a raised brain natriuretic peptide or $\mathrm{N}$-terminal pro-brain natriuretic peptide within the past 30 days

- Intervention and comparison: Spironolactone or placebo

- Outcome: The primary outcome is a composite of cardiovascular mortality, aborted cardiac arrest, and admission to hospital because of heart failure.

\section{What should we do in the light of the uncertainty?}

Despite the uncertainties in the current evidence base, how should patients with heart failure with preserved ejection fraction be treated? Firstly, a diuretic should be used to relieve limiting heart failure symptoms, such as pulmonary congestion. Secondly, an ACE inhibitor and a $\beta$ blocker should be considered, particularly when there are other compelling indications for their uses (such as coronary artery disease, hypertension, and diabetes mellitus). Thirdly, if atrial fibrillation is present, digoxin may be added for rate control. Lastly, treatments of comorbidities commonly found in patients with HF-PEF, such as anticoagulation for atrial fibrillation, should be pursued. These recommendations are consistent with those in the current NICE guideline. ${ }^{6}$

In sum, we are not convinced by the current evidence for any of the drug treatments in HF-PEF in reducing morbidity or mortality in this population. However, given the benefits of various drugs for allied conditions and in HF-REF, we believe that extrapolations of evidence from related areas and consideration of the limited data from trials in the specific populations together can help to determine the most appropriate treatment for patients with HF-PEF. We would not disagree, however, with any physician who wishes to withhold any of the treatments that we recommend.

Contributors: SY conceived the idea for the article. PJ and RMcK conducted the literature review. All authors drafted the manuscript. SY is the guarantor.

Competing interests: All authors have completed the Unified Competing Interest form at www.icmje.org/coi_disclosure.pdf (available on request from the corresponding author) and declare that (1) none of the authors have support for the submitted work from anyone; (2) SY has received grants and honorariums from Boehringer-Ingelheim, AstraZeneca, Bristol Myers

Squibb, Merck, Sanofi-Aventis, and Novartis. RMcK has received grants and

honorariums from AstraZeneca, Bristol Myers Squibb, and Sanofi-Aventis; (3) 
bmj.com archive

Previous articles in this

series

- How beneficial is

surgery for cervical

radiculopathy and

myelopathy? (BM)

2010;341:c3108)

- Does avoidance

of peanuts in early

life reduce the risk of

peanut allergy? (BM)

2010;340:c424)

- Should we use

bath emollients for

atopic eczema? (BM)

2009;339:b4273) none of the authors' spouses, partners, or children have financial relationships that may be relevant to the submitted work; and (4) none of the authors have non-financial interests that may be relevant to the submitted work.

Provenance and peer review: Commissioned; externally peer reviewed.

1 Hogg K, Swedberg K, McMurray J. Heart failure with preserved left ventricular systolic function; epidemiology, clinical characteristics, and prognosis. J Am Coll Cardiol 2004;43:317-27.

2 Bhatia RS, Tu JV, Lee DS, Austin PC, Fang J, Haouzi A, et al. Outcome of heart failure with preserved ejection fraction in a population-based study. NEngl/ Med 2006;355:260-9.

3 Owan TE, Hodge DO, Herges RM, Jacobsen SJ, Roger VL, Redfield MM. Trends in prevalence and outcome of heart failure with preserved ejection fraction. N Engl / Med 2006;355:251-9.

4 Fonarow GC, Stough WG, Abraham WT, Albert NM, Gheorghiade M, Greenberg BH, et al. Characteristics, treatments, and outcomes of patients with preserved systolic function hospitalized for heart failure: a report from the OPTIMIZE-HF Registry. J Am Coll Cardiol 2007;50:768-77

5 Paulus WJ, Tschope C, Sanderson JE, Rusconi C, FlachskampfFA, Rademakers FE, et al. How to diagnose diastolic heart failure: a consensus statement on the diagnosis of heart failure with normal left ventricular ejection fraction by the Heart Failure and Echocardiography Associations of the European Society of Cardiology. Eur Heart J 2007;28:2539-50.

6 National Institute for Health and Clinical Excellence. Chronic heart failure: the management of adults with chronic heart failure in primary and secondary care (partial update). (Clinical guideline 108). 2010. www.nice.org.uk/CG108.

7 Cleland JG, Tendera M, Adamus J, Freemantle N, Polonski L, Taylor J. The perindopril in elderly people with chronic heart failure (PEP-CHF) study. Eur Heart/2006;27:2338-45.
8 Yusuf S, Pfeffer MA, Swedberg K, Granger CB, Held P, McMurray JJ, et al. Effects of candesartan in patients with chronic heart failure and preserved left-ventricular ejection fraction: the CHARM-Preserved Trial. Lancet 2003;362:777-81.

9 O'Meara E, Solomon S, McMurray J, Pfeffer M, Yusuf S, Michelson E, et al. Effect of candesartan on New York Heart Association functional class. Results of the candesartan in heart failure: assessment of reduction in mortality and morbidity (CHARM) programme. Eur Heart) 2004;25:1920-6.

10 Massie BM, Carson PE, McMurray JJ, Komajda M, McKelvie R, Zile MR, et al. Irbesartan in patients with heart failure and preserved ejection fraction. NEngl J Med 2008;359:2456-67.

11 Ahmed A, Rich MW, Fleg JL, Zile MR, Young JB, Kitzman DW, et al. Effects of digoxin on morbidity and mortality in diastolic heart failure: the ancillary digitalis investigation group trial. Circulation 2006;114:397-403.

12 Digitalis Investigation Group. The effect of digoxin on mortality and morbidity in patients with heart failure. N Engl/ Med 1997:336:525-33.

13 Flather MD, Shibata MC, Coats AJ, Van Veldhuisen DJ, Parkhomenko A, Borbola J, et al. Randomized trial to determine the effect of nebivolol on mortality and cardiovascular hospital admission in elderly patients with heart failure (SENIORS). Eur Heart/ 2005;26:215-25.

14 Dagenais GR, Pogue J, Fox K, Simoons ML, Yusuf S. Angiotensinconverting-enzyme inhibitors in stable vascular disease without left ventricular systolic dysfunction or heart failure: a combined analysis of three trials. Lancet 2006;368:581-8.

15 Cleland JG, Coletta AP, Yassin A, Buga L, Torabi A, Clark AL. Clinical trials update from the European Society of Cardiology Meeting 2009: AAA, RELY, PROTECT, ACTIVE-I, European CRT survey, German pre-SCD II registry, and MADIT-CRT. Eur J Heart Fail 2009;11:1214-9.

16 Bangalore S, Wild D, Parkar S, Kukin M, Messerli FH. Beta-blockers for primary prevention of heart failure in patients with hypertension insights from a meta-analysis. J Am Coll Cardiol 2008;52:1062-72.

\title{
EASILY MISSED? Foreign body inhalation in children
}

\author{
Kay Wang, ${ }^{1}$ Anthony Harnden, ${ }^{1}$ Anne Thomson ${ }^{2}$
}

${ }^{1}$ Department of Primary Health Care, University of Oxford, Oxford OX37LF

${ }^{2}$ ohn Radcliffe Hospital, Oxford OX39DU

Correspondence to: A Thomson

anne.thomson@orh.nhs.uk

Cite this as: BMJ 2010;341:C3924 doi: 10.1136/bmi.c3924

This is a series of occasional articles highlighting conditions that may be more common than many doctors realise or may be missed at first presentation. The series advisers are Anthony Harnden, university lecturer in general practice, Department of Primary Health Care, University of Oxford, and Richard Lehman, general practitioner, Banbury. If you would like to suggest a topic for this series please email us (easilymissed.bmj@bmjgroup. com).
Children, especially toddlers, tend to place objects in their mouths while exploring their environment. They are therefore at increased risk of inhaling foreign bodies, which may become lodged in the tracheobronchial system.

\section{Why is it missed?}

Diagnosis of an inhaled foreign body was delayed by more than a week in $29 \%$ of cases and by more than 30 days in $10 \%$ in a retrospective analysis of 1015 cases. ${ }^{4} \mathrm{~A}$ witnessed choking event is the most important factor in pinpointing an early (within 24 hours) diagnosis of foreign body inhalation. ${ }^{5}$ However, a consecutive series of 142 children attending a hospital emergency department with a history suggesting foreign body inhalation found that such events are not reported in 1 in 6 (10/61) confirmed cases. ${ }^{6}$ Parents or carers may not witness or remember choking episodes, and children may not disclose a history of choking or of inhaling foreign bodies because of limited speech, fear or embarrassment.

Clinicians may fail to consider the diagnosis of an inhaled foreign body if the child has no symptoms at presentation or presents with prolonged or atypical symptoms, especially when physical examination and chest radiograph findings are normal. ${ }^{7}$ Inhaled objects that do not cause an intense inflammatory response (plastic toys, for example) or that result in only partial airway occlusion are the most difficult to detect.

\section{KEY POINTS}

A diagnosis of foreign body inhalation should be considered in any child in whom a witnessed choking episode is reported

Clinicians should specifically ask about previous choking episodes in children presenting with persistent cough or other respiratory symptoms

Bronchoscopy is recommended in children with a suggestive history together with symptoms, signs, or chest radiograph findings consistent with foreign body inhalation; an index of suspicion should be maintained in children with a suggestive history only

\section{Why does it matter?}

Delayed diagnosis of an inhaled foreign body can result in complete airway obstruction, which may be fatal. ${ }^{3}$ The rate of serious acute complications (including pneumonia, pneumothorax, and subglottic oedema) is 2.5 times higher when diagnosed more than 24 hours after inhalation (67\%) than when diagnosed within 24 hours $(27 \%){ }^{8}$ Long term complication rates are also higher if diagnosis is delayed. In one follow-up study almost 30\% (6/21) of children in whom foreign body removal was delayed by more than one week developed a chronic persistent cough (mean duration of follow-up 2.05 years). ${ }^{9}$ Recurrent pneumonia, lung abscesses, and bronchiectasis can also develop if foreign bodies remain in place for many weeks. $^{10}$ 


\section{CASE SCENARIO}

A 2 year old boy presented to his general practitioner with a two week history of a dry, persistent cough. His mother recalled an episode two weeks ago when he had a violent coughing fit while eating nuts and raisins. She took him to the nearest hospital emergency department, but he was discharged a few hours later after a normal physical examination and normal chest radiograph. Nevertheless, this history of persistent cough following a choking episode should raise concern about possible foreign body inhalation.

\section{HOW COMMON IS IT?}

During 2008-9, just over 300 hospital admissions in England were due to foreign body inhalation in children up to 14 years of age ${ }^{1}$

Worldwide, $55 \%$ of children who have inhaled foreign bodies are between 1 and 3 years of age and $7-10 \%$ are under 1 year of age ${ }^{2}$

In the United States, foreign body inhalation accounts for $7 \%$ of accidental deaths in children under 4 years of age ${ }^{3}$

\begin{tabular}{|c|c|c|c|c|}
\hline \multicolumn{5}{|c|}{ Diagnostic value of clinical findings in foreign body inhalation } \\
\hline Findings & $\begin{array}{l}\text { Sensitivity } \\
(\%)\end{array}$ & $\begin{array}{l}\text { Specificity } \\
(\%)\end{array}$ & $\begin{array}{l}\text { Positive predictive } \\
\text { value (\%) }\end{array}$ & $\begin{array}{l}\text { Negative predictive } \\
\text { value (\%) }\end{array}$ \\
\hline History of witnessed choking event ${ }^{6}$ & 92 & 32 & 50 & 84 \\
\hline Cough $^{6}$ & 92 & 28 & 49 & 82 \\
\hline Wheeze $^{12}$ & 88 & 28 & 76 & 47 \\
\hline Dyspnoea $^{12}$ & 18 & 74 & 64 & 26 \\
\hline Localised decreased lung sounds ${ }^{6}$ & 57 & 85 & 74 & 73 \\
\hline
\end{tabular}

\section{bmj.com archive}

Previous articles in this series

\section{- Ectopic pregnancy} (BMJ 2010;341:C3770)

- Testicular torsion (BMJ

2010;341:c3213)

- Bronchiectasis (BM)

2010;341:C2766)

- Endometriosis (BMJ

2010;340:c2661)

- Biliary atresia (BM)

2010;340:c2383)

\section{How is it diagnosed?}

\section{Clinical features}

The table lists the sensitivities, specificities, and positive and negative predictive values of clinical features associated with foreign body inhalation in children. In a 10 year chart review, 77 out of 135 children (57\%) showed the classic triad of coughing or choking, wheezing, and unilateral reduced breath sounds. ${ }^{7}$ Wheeze is present when there is partial obstruction of an airway. Persistent cough, wheeze, sputum production, and dyspnoea may develop in children in whom diagnosis is delayed by more than one month. ${ }^{11}$

\section{Investigations}

Chest radiograph findings compatible with an inhaled foreign body include air trapping, atelectasis, and pneumothorax. After several days, radiographic evidence of pneumonia may also be present. However, none of these findings are pathognomonic for foreign body inhalation. ${ }^{13}$ In addition, more than three quarters of inhaled foreign bodies are radiolucent and will therefore not show on chest radiographs. ${ }^{14}$

Definitive diagnosis of foreign body inhalation is by endoscopic evaluation. The most appropriate first line procedure depends on the likelihood of a foreign body being present. Where there is near certainty, rigid bronchoscopy under general anaesthesia is the investigation of choice, as the object can be detected and removed in one procedure. ${ }^{6}$ In cases where clinical and radiographic findings are equivocal, flexible bronchoscopy under sedation and local anaesthesia can be useful in confirming the presence and exact site of the item. ${ }^{2}$ Subsequent removal is by rigid bronchoscopy under general anaesthesia.

\section{How is it managed?}

Bronchoscopy is recommended when children have a suggestive history together with symptoms, signs, or chest radiograph findings consistent with foreign body inhalation. Children with a suggestive history but normal clinical and chest radiograph findings should be monitored carefully for symptoms and signs of foreign body inhalation. ${ }^{6}$ Given the considerable mortality and morbidity associated with foreign body inhalation in children, the importance of preventive measures needs to be emphasised to parents and carers.

Contributors: $\mathrm{KW}$ wrote the first draft of the article, which was modified by $\mathrm{AH}$ and AT. All authors agreed on the final draft. AT is guarantor.

Funding: KW's post is funded by the National Institute for Health Research.

Competing interests: AT has given a presentation for Kayonesse Conferences on cough and wheeze in children for which financial payment was received by AT's academic institution.

Provenance and peer review: Not commissioned; externally peer reviewed.

1 Hospital Episode Statistics. External cause, 2008-9. http://www. hesonline.nhs.uk/Ease/servlet/ContentServer?sitelD=1937\&catego rylD=211.

2 Righini CA, Morel N, Karkas A, ReytE, Ferretti K, Pin I, et al. What is the diagnostic value of flexible bronchoscopy in the initial investigation of children with suspected foreign body aspiration? Int J Pediatr Otorhinolaryngol 2007;71:1383-90.

3 Mantor PC, Tuggle DW, Tunell WP. An appropriate negative bronchoscopy rate in suspected foreign body aspiration. Am / Surg 1989;158:622-4.

4 Saki N, Nikakhlagh S, Rahim F, Abshirini H. Foreign body aspirations in infancy: a 20-year experience. Int/Med Sci 2009:6:322-8.

5 Chiu C-Y, Wong K-S, Lai S-H, Hsia S-H, Wu C-T. Factors predicting early diagnosis of foreign body aspiration in children. Pediatr Emerg Care 2005;21:161-4.

6 Cohen S, Avital A, Godfrey S, Gross M, Kerem E, Springer C. Suspected foreign body inhalation in children: what are the indications for bronchoscopy? J Pediatr 2009;155:276-80.

7 Tan HKK, Brown K, McGill T, Kenna MA, Lund DP, Healy GB. Airway foreign bodies (FB): a 10-year review. IntJ Pediatr Otorhinolaryngol 2000;56:91-9.

8 Esclamado RM, Richardson MA. Laryngotracheal foreign bodies in children: a comparison with bronchial foreign bodies. Am J Dis Child 1987;141:259-62.

9 Davies H, Gordon I, Matthew DJ, Helms P, Kenney IJ, Lutkin JE, et al. Long term follow up after inhalation of foreign bodies. Arch Dis Child 1990;65:619-21.

10 Abdulmajid OA, Ebeid AM, Motaweh MM, Kleibo IS. Aspirated foreign bodies in the tracheobronchial tree: report of 250 cases. Thorax 1976;31:635-40.

11 Karakoc F, Cakir E, Ersu R, Uyan ZS, Colak B, Karadag B, et al. Late diagnosis of foreign body aspiration in children with chronic respiratory symptoms. Int J Pediatr Otorhinolaryngol 2007;71:241-6.

12 Kiyan G, Gocmen B, Tugtepe H, Karakoc F, Dagli E, Dagli TE. Foreign body aspiration in children: The value of diagnostic criteria. Int/ Pediatr Otorhinolaryngol 2009;73:963-7.

13 Svedstrom E, Puhakka H, Kero P. How accurate is chest radiography in the diagnosis of tracheobronchial foreign bodies in children? Pediatr Radiol 1989;19:520-2.

14 SersarSI, RizkWH, Bilal M, El Diasty MM, Eltantawy TA, Abdelhakam BB, et al. Inhaled foreign bodies: presentation, management and value of history and plain chest radiography in delayed presentation. Otolaryngol Head Neck Surg 2006;134:92-9.

Accepted: 22 June 2010

\section{Endpiece Explain it to your grandmother}

You do not really understand something unless you can explain it to your grandmother.

Albert Einstein (1879-1955)

Submitted by Alan Cordey, foundation year 1 doctor,

Bristol Royal Infirmary, Bristol

Cite this as: BMJ 2010;341:C3723 